\title{
Analytical Methods for Assessment of Temporal Changes in Heart Function
}

\author{
Yoshio Kan $^{*}$, Koji Kashihara ${ }^{\dagger}$
}

\begin{abstract}
Quick and automatic detection of abnormal signals in electroencephalogram (ECG) can help cardiovascular patients. We firstly focused on the judgment of ST depression as an abnormal ECG signal. The optimal threshold was explored by the modified cross-validation analysis based on a correlation coefficient between ECG data on the ST depression as a template and the other disease (i.e., ventricular fibrillation or abnormal $\mathrm{T}$ waves). The optimal threshold of the correlation coefficient was around 0.8 . The calculated threshold was little affected by the type of linear or spline interpolation and data length (i.e., 100, 200, and 300 points for the normalization). These results could reduce the computation time in online analysis of $e$-healthcare applications. Next, we assessed the temporal change in individual's heart function during the advanced trail making test (ATMT). The heart rate variability (HRV) analysis was performed in the time or frequency domain, and it was able to reflect healthy and unhealthy conditions during the ATMT. This result will be significantly affected by the activity of the autonomic nervous system. The indices for the HRV could be applied to a home healthcare system to find potential patients from daily temporal changes in heart function.
\end{abstract}

Keywords: cardiovascular diseases, fatigue, heart rate, template matching.

\section{Introduction}

Today heart disease has been becoming one of the main causes of death in the world [1]. Electroencephalogram (ECG) is usually measured to check abnormal waves of cardiovascular patients. In a clinical situation, it is crucial to quickly notice cardiac abnormalities from ECG data (e.g., sudden arrhythmia, long QT syndrome, and ST depression [2][3]). In special, Holter ECG monitoring is a simple and effective method to diagnose cardiac patients. The early detection of ECG abnormalities can avoid falling into severe disease. However, clinical

* Graduate School of Advanced Technology and Science, Tokushima University, Tokushima, Japan

$\dagger$ Institute of Technology and Science, Tokushima University, Tokushima, Japan 
doctors may be unable to check all of the previously recorded long-term data in each patient. If the cardiovascular abnormalities are automatically detected from the ECG data, using a simple computer system or a software application, the analytical results could help medical doctors and find potential patients. Therefore, the first objective of this study (Study 1: Automatic detection of abnormal ECG data) was to investigate an efficient analytical method to automatically catch cardiovascular disease from clinical ECG data. Although the accuracy of automatic detection in the abnormal ECG signals has been increased [4], the final judgement of diseases will depend on medical doctors. The sensitivity of the automatic ECG analysis may be also increased to prevent oversight of disease, as the first priority (e.g., a school medical examination requiring the assessment of many students at once). Such a medical examination will increase the burden of medical doctors because of rechecking the ECG data; it is required to improve the accuracy of the automatic detection. In special, it is a crucial task to quickly and automatically find the ST changes associated with heart diseases [5]. We therefore focused on the analytical method to automatically find the ST segment abnormalities as well as the typical heart diseases such as ventricular fibrillation (VF) and abnormal $\mathrm{T}$ waves.

In a cardiac patient who does not need to enter hospital, a Holter ECG recording can monitor the day-to-day function of the heart [6]. Even in healthy people, such a monitoring system has a possibility to find potential cardiac abnormalities from daily ECG signals. If individual ECG waves are measured for a long period and accumulated data are compared each other, a slight signal change unseen previously may be detected. Such change has a possibility to show a sign of a coming heart disease, even within a normal wave range of ECG. Even if he/she looks healthy, the sign of a heart abnormality including the fatigue or stress may be abstracted from the slight change in individually collected ECG data. A simple analytical method and system will be efficient for daily checking of temporal changes in the heart function which cannot be found by one time inspection of ECG. Therefore, the second objective of this study (Study 2: Temporal changes in heart function) was to analyze temporal changes in the heart function (e.g., RR intervals) based on daily physical conditions. We focused on the RR intervals of ECG and performed the heart rate variability (HRV) analysis in the time [7] and frequency [8] domains during the advanced trail making test (ATMT) which can induce acute fatigue [9].

\section{Automatic Detection of Abnormal ECG Data}

\subsection{Related Works}

Previous studies have described the techniques and algorithms for the automatic detection of ECG data (e.g., [10][11]). The frequency or time-frequency analysis makes it possible to facilitate the accuracy of the automatic detection because of the signal feature extraction with noise reduction; the morphological technique [12], template matching [13], fuzzy logic [14], and neural networks [15] have been applied to identify abnormal ECG patterns. However, the methods for the automatic detection should be further altered to increase the accuracy. In special, it may be difficult to catch the ST segment or ST-T complex changes [16][17]. The template matching technique may be easier than the other methods requiring the detailed parameter settings or mathematical models to detect specific patterns. We, therefore, tested the combination of the template matching with correlation coefficients [18] and the modified cross-validation analysis to categorize heart disease including the ST depression. The wire-less ECG system [19] based on the above technique could be useful for the early detection of various diseases. 


\subsection{Analytical Methods}

(1) ECG dataset: Fifty ECG data (Fig. 1) were applied to this study: (a) 25 data of ST depression, (b) 9 data immediately before VF, (c) 8 data during VF, and (d) 8 data of abnormal $\mathrm{T}$ waves. The ECG dataset was provided from the website of PhysioNet (http://www.physionet.org/), a database of the biomedical signal waveform. The ECG waveforms were categorized into Group $A$ [(a) ST depression] and Group $B$ [(b), (c), and (d) the other diseases]. From Group $A$, we created template data for automatic detection of the ST depression. The correlation coefficient between the template in Group $A$ and the test data in Group $B$ was computed to classify the ST depression and other diseases.

Group A

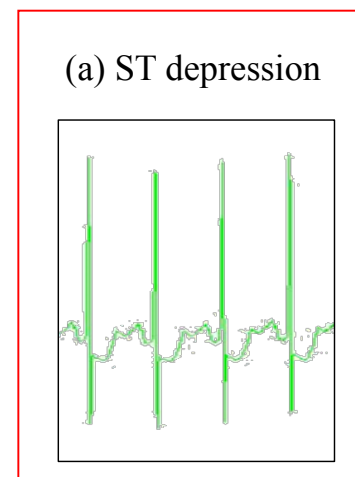

25 data
Group B

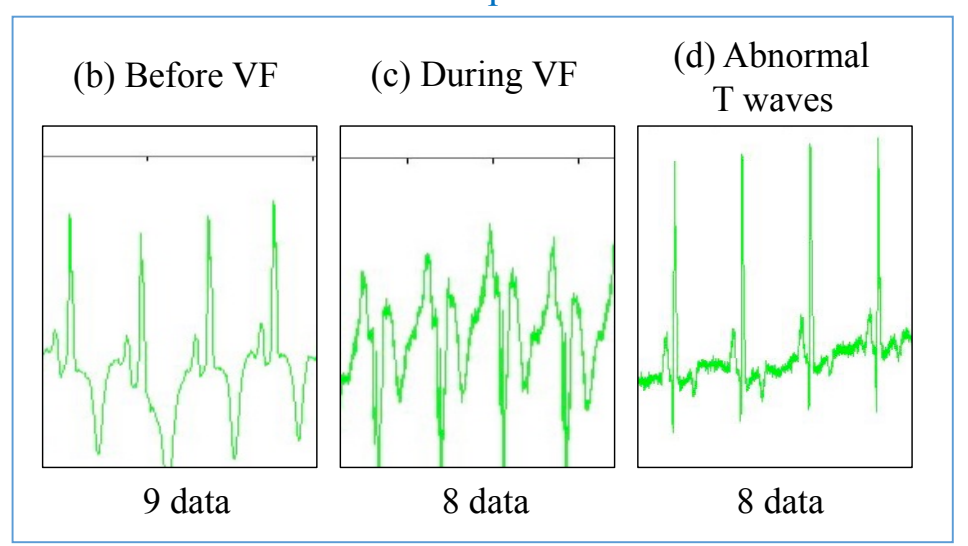

Figure 1: Examples of abnormal ECG waves: (a) ST depression, (b) before and (c) during $\mathrm{VF}$, and (d) abnormal $\mathrm{T}$ waves.

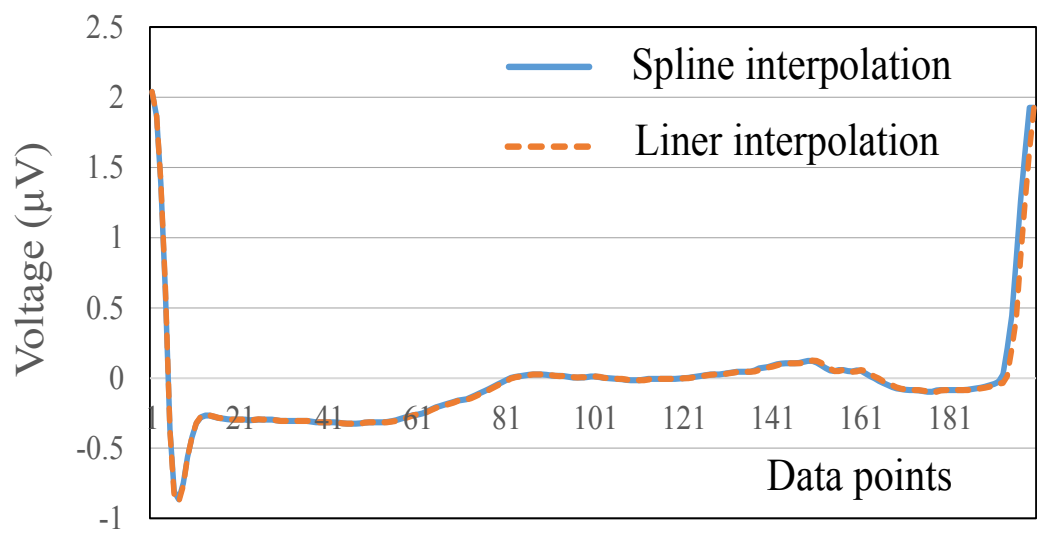

Figure 2: Linear and spline interpolations for the normalization of ECG waveforms.

(2) Preprocessing of ECG data: One-beat signal was automatically detected by searching for the R waves (i.e., peak values of ECG). The window size of the extracted single waveform was varied among all ECG data because it depends on the heartbeat; it was normalized by the linear, spline, and Lagrange interpolations every 200 data points because actual data length of ECG was the range from 150 to 250 data points. To confirm the accuracy of the window size, other template length (100 or 300 data points) was also assessed in the spline 
interpolation. Here, we selected the spline interpolation because there are no difference between the results of the spline and linear interpolations (200 data points), as shown in Fig. 2. The slight difference observed between the interpolations could be allowed to carry out the automatic detection because the artificial noise or error on ECG signals is generally included (e.g., around 10\% [20]). However, the Lagrange interpolation was excluded from further analysis owing to the insufficient interpolation depending on data points.

(3) Modified cross-validation analysis: A template waveform for ST depression was prepared for the modified cross-validation analysis, using the dataset of Group $A$ (i.e., 25 waveforms). The template was created from the average of 24 waveforms (the mean template) in Group $A$; the remaining data (i.e., a waveform in Group $A$ ) was applied to the cross-validation analysis. Therefore, twenty five patterns of the different template waveform were created because the total number of ECG data was 25 in Group $A$. The correlation coefficient was then computed from the mean template and the remaining waveform in Group $A$. We also evaluated the correlation coefficient between the mean template in Group $A$ (i.e., ST depression) and one of the wave patterns in Group $B$ (i.e., other heart diseases). Here, the mean template in Group $A$ was randomly changed.

\subsection{Analytical Results and Discussion}

Table 1 shows the correlation coefficient between the ECG data on the template and cardiovascular diseases in the case of spline or linear interpolation. For the spline interpolation (200 data points of the template length), the correlation coefficient between the template and ST depression was ranged from 0.86 to 0.97 . The correlation coefficient in the case of the ST depression was close to that in the abnormal $\mathrm{T}$ waves. However, the correlation coefficient was greatly lower in the cases before and during VF than in the ST depression. For the liner interpolation (200 data points), the correlation coefficient between the template and ST depression showed a higher value ( 0.90 to 0.97 ), compared with that in the abnormal $\mathrm{T}$ waves (0.58 to 0.67$)$. In contrast, the correlation coefficient before or during VF (0.20 to 0.59 ) was greatly lower than that in the ST depression.

Table 1: Correlation Coefficients Estimated by Spline or Linear Interpolation in Each Template Size

\begin{tabular}{|c|c|c|c|c|}
\hline \multirow{3}{*}{ ECG data } & \multicolumn{4}{|c|}{ Number of Data Points } \\
\hline & \multirow{2}{*}{$\begin{array}{c}100 \\
\text { (Spline) }\end{array}$} & \multicolumn{2}{|c|}{200} & \multirow{2}{*}{$\begin{array}{c}300 \\
\text { (Spline) }\end{array}$} \\
\hline & & Spline & Linear & \\
\hline $\begin{array}{c}\text { ST depression } \\
\text { (Group } A: 25 \text { data) }\end{array}$ & $\begin{array}{l}0.94 \pm 0.03 \\
(0.88,0.97)\end{array}$ & $\begin{array}{l}0.92 \pm 0.03 \\
(0.86,0.97)\end{array}$ & $\begin{array}{l}0.94 \pm 0.02 \\
(0.90,0.97)\end{array}$ & $\begin{array}{l}0.92 \pm 0.03 \\
(0.85,0.97)\end{array}$ \\
\hline $\begin{array}{c}\text { Before VF } \\
\text { (Group } B: 9 \text { data) }\end{array}$ & $\begin{array}{l}0.43 \pm 0.04 \\
(0.39,0.48)\end{array}$ & $\begin{array}{l}0.37 \pm 0.04 \\
(0.33,0.42)\end{array}$ & $\begin{array}{l}0.40 \pm 0.05 \\
(0.32,0.48)\end{array}$ & $\begin{array}{l}0.35 \pm 0.04 \\
(0.30,0.40)\end{array}$ \\
\hline $\begin{array}{c}\text { During VF } \\
\text { (Group } B: 8 \text { data) }\end{array}$ & $\begin{array}{l}0.30 \pm 0.05 \\
(0.20,0.35)\end{array}$ & $\begin{array}{l}0.27 \pm 0.05 \\
(0.17,0.33)\end{array}$ & $\begin{array}{l}0.33 \pm 0.11 \\
(0.20,0.59)\end{array}$ & $\begin{array}{c}0.22 \pm 0.11 \\
(-0.02,0.32)\end{array}$ \\
\hline $\begin{array}{c}\text { Abnormal T waves } \\
\text { (Group } B: 8 \text { data) }\end{array}$ & $\begin{array}{l}0.76 \pm 0.02 \\
(0.73,0.78)\end{array}$ & $\begin{array}{l}0.69 \pm 0.03 \\
(0.66,0.72)\end{array}$ & $\begin{array}{l}0.63 \pm 0.03 \\
(0.58,0.67)\end{array}$ & $\begin{array}{c}0.66 \pm 0.03 \\
(0.64,0.70)\end{array}$ \\
\hline
\end{tabular}

Average \pm S.D. $(\min , \max )$. 
Table 1 also summarizes the correlation coefficient between the ST depression and other diseases under the different template length (i.e., 100 and 300 data points) with the spline interpolation. For the cases of the VF and abnormal T waves, the shorter length of the template (i.e., 100 data points) resulted in the increased correlation coefficient; on the other hand, the result of 300 data points had the tendency for the decreased correlation coefficient. However, the correlation coefficient under the ST depression was the almost same as that in the 200 data points in the template, and the number of data points for the normalization did not greatly affect the correlation coefficient values of the ECG waveforms. Therefore, the accuracy to detect the abnormal ECG will be kept even when the data points are set between 100 and 300 .

Figure 3 shows the correct matching number under the threshold value to automatically judge the ST depression of a target ECG data, under the template length of 200 data points in the spline interpolation. The threshold of the correlation coefficient ranged from 0.72 (i.e., the maximum value in Group $B$ ) to 0.86 (i.e., the minimum value in Group $A$ ). To classify the data between Groups $A$ and $B$, the optimal threshold with the accuracy of $100 \%$ (i.e., the correct matching number $=25$ ) was at around 0.8 (i.e., the red bar in Fig. 3). In the linear interpolation between two points (200 data points), the threshold value was between 0.67 and 0.90 . For 100 data points of a template length (spline interpolation), the threshold of the correlation coefficient ranged from 0.78 to 0.88 ; it was between 0.70 and 0.85 in 300 data points. Therefore, when the threshold of the correlation coefficient in all the cases is set at 0.8 , the categorization accuracy can show $100 \%$. The clinical ECG signals could be accurately classified into the ST depression and other diseases, by setting the optimal threshold based on the correlation coefficient; the identification rate of this method may be higher than the general ones (e.g., [13][17]). This approach may be able to automatically detect potential abnormalities from daily ECG signals [18].

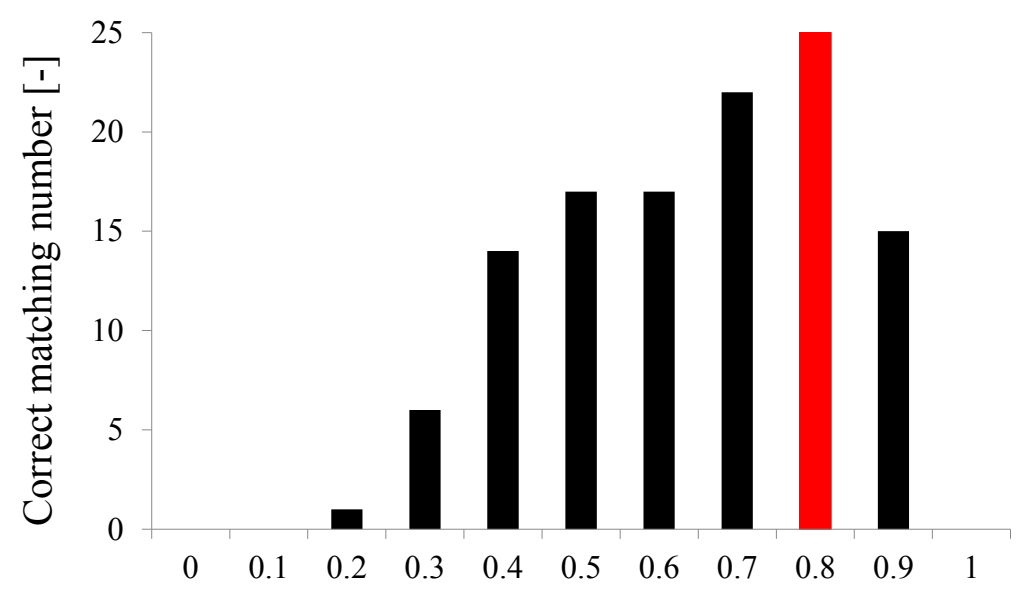

Correction coefficient for the threshold value [-]

Figure 3: Relationship between the correct matching number and the correlation coefficient as the threshold to automatically detect the ST depression. 


\section{Temporal Changes in Heart Function}

\subsection{Related Works}

The ATMT can estimate the change in the daily level of stress or fatigue [21] affecting cardiac rhythms [22]; furthermore, the central nervous system modulates the feature of ECG signals during the ATMT [23]. However, the detailed individual characteristics including unusual conditions remain unknown. Therefore, in each individual, we performed the HRV analysis [7][8] on the temporal ECG changes during the ATMT. A wireless or wearable system [19] based on the proposed analysis could detect abnormalities of daily heart function.

\subsection{Experimental Methods}

(1) Subjects: Participants were four male students (mean age \pm S.D.: $22.5 \pm 1.7$ years in Subjects $A$ to $D$ ). All participants had normal or corrected-to-normal vision. The experimental procedure was performed in accordance with the declaration of Helsinki. A detailed description of the experimental procedure, and written informed consent was obtained from all participants.

Each subject had the experiment three times; the experimental interval was about one week. The experimenter firstly confirmed the health condition of the subjects. Subjects $A$ and $B$ were healthy conditions in all experiments. However, Subject $C$ had signs of a slight cold in the 'second' experiment; Subject $D$ complained of fatigue in the 'third' experiment.

(2) Procedures: The visual stimuli for the ATMT were presented at eye level against a screen monitor in a quiet experimental room. Because the participants were required to become familiar with the answer method of the ATMT, they sufficiently practiced to perform actual tasks before a few days of the first experimental day (about 10 to 20 trials).

Figure 4(a) shows the experimental procedure for the ATMT with the measurement of RR intervals. After a resting period for 5 min, a subject performed the ATMT (12 trials in total), followed by a recovery period for $5 \mathrm{~min}$. First, the numbers from 1 to 25 were randomly displayed [Fig. 4(b)], and the subject was required to search for the smallest number (e.g., 1 in the first choice) and to press a mouse button on the target number. After selecting the target, the next number of the largest one was added (e.g., 2 to 26 in the second choice); all numbers were randomly assigned on a display, without the previously selected target number. When the final target number 25 was chosen, the single trial was finished. The next trial was started immediately after a trial, and twelve trials were performed in total. Therefore, the completion time for this task was different every participant. The RR intervals (RS800CX, Polar Co.) for the HRV analysis were continuously recorded during the experiment. 
(a) Experimental procedure

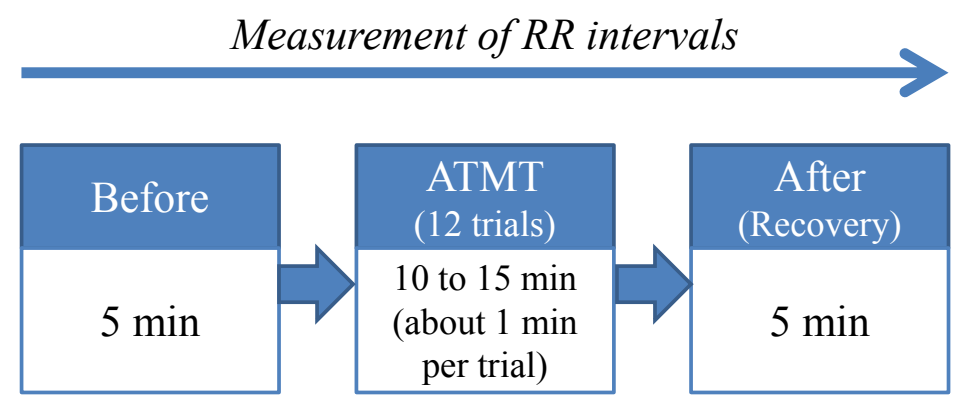

(b) Display of ATMT

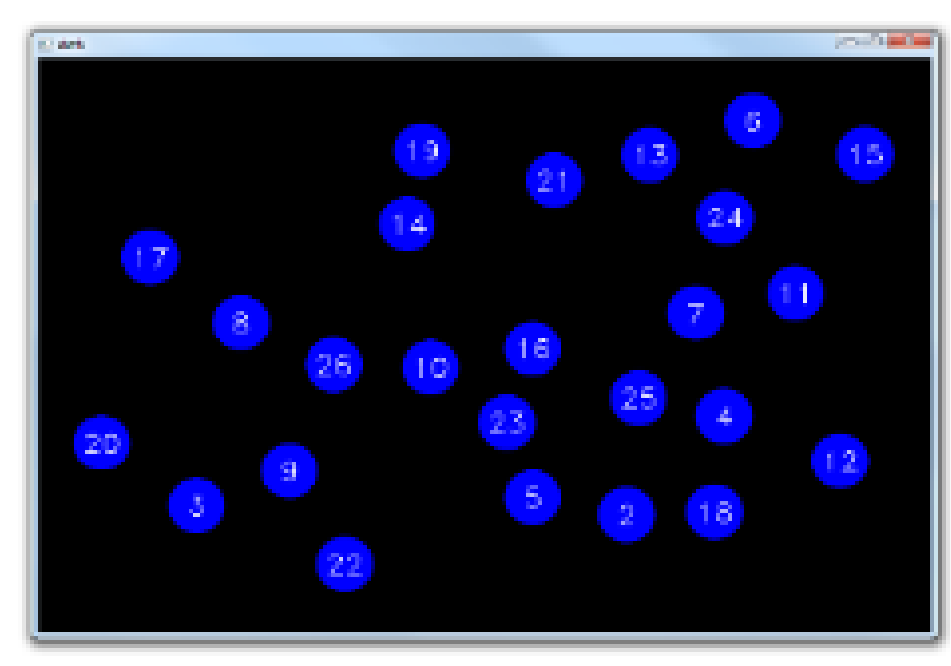

Figure 4: (a) The experimental procedure and (b) an example of the ATMT (the smallest number with the blue circle, the target for this task).

(3) Analytical methods: Reaction times were recorded during the ATMT; the multiple trials of the first (i.e., 1 to 6 trials) or second (i.e., 7 to 12 trials) half were averaged in each experiment. The RR intervals were divided into four periods: five min before, during (the first and the latter), and after the ATMT. The RR intervals were analyzed under the time and frequency domains. The Poincaré plot [Fig. 5(a)] was used for the time domain HRV analysis [7]. The Poincaré plot graphically indicates the correlation between consecutive RR intervals $[x$ and $y$ axes: $\operatorname{RR}(n)$ and $\mathrm{RR}(n+1)]$. The standard deviations of the points (i.e., an ellipse) were defined as $\mathrm{SD}_{1}$ and $\mathrm{SD}_{2}$. The $\mathrm{SD}_{1}$ means the short variation of $\mathrm{RR}$ intervals reflecting the effects of respiration on vagal drive. The $\mathrm{SD}_{2}$ means the long variation reflecting other heart rate changes. Low values of the $\mathrm{SD}_{1}$ and $\mathrm{SD}_{2}$ will indicate high stress [24].

For the frequency domain analysis, the fast Fourier transform (FFT) on 512 data points ( 5 min) was applied to time series of the RR interval; the hamming window with $50 \%$ overlap was used for the FFT analysis. The power spectrum density was then calculated [Fig. 5(b)]. The target frequency band for the HRV analysis is divided into two components: the low frequency (LF) component (i.e., 0.04 to $0.15 \mathrm{~Hz}$ ) and the high frequency (HF) component 
(i.e., 0.15 to $0.4 \mathrm{~Hz}$ ). The LF component reflects the sympathetic and parasympathetic activities. The HF component mainly reflects the parasympathetic activity (e.g., the inhibited vagal tone during inspiration). Natural respiration was selected for this experiment. The $\mathrm{LF} / \mathrm{HF}$ ratio was used as an index of sympathovagal balance [8].

(a) Poincaré plot

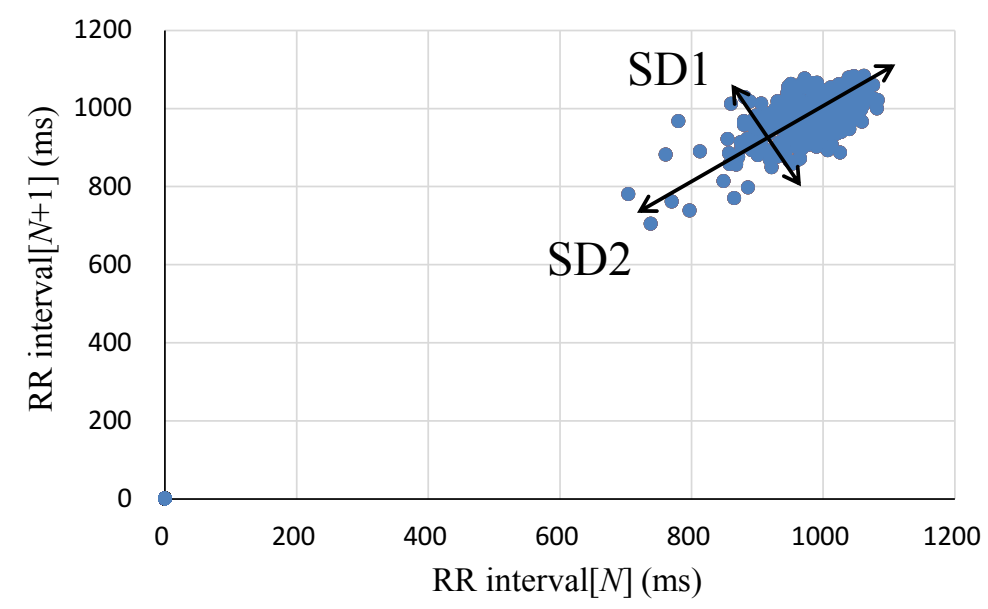

(b) Power spectrum analysis

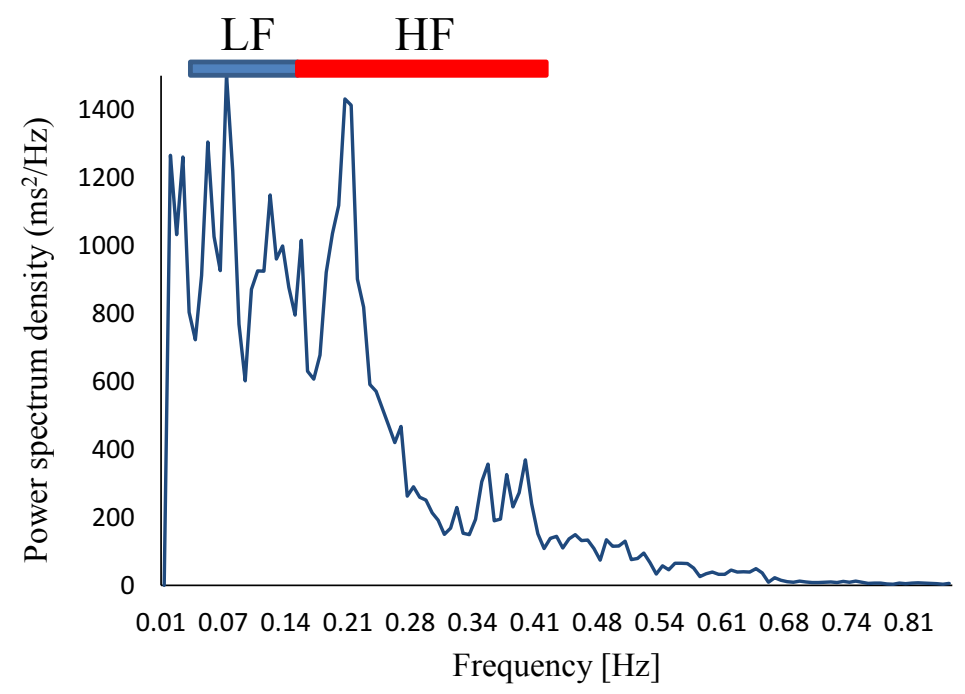

Figure 5: Examples of (a) the Poincaré plot $\left(\mathrm{SD}_{1}\right.$ and $\left.\mathrm{SD}_{2}\right)$ and (b) the power spectrum analysis (the blue bar: the frequency band of the LF component; the red bar: that of the HF component).

For the evaluation index for the HRV analysis, we calculated the grand average across all experiments. The Friedman test, followed by the Tukey's post hoc test, was then performed between the experimental periods. Although the statistical data included the results of different experimental days every subject, we considered them as the same weight. For the reaction times, the Wilcoxon signed rank sum test was performed between the first and second half data. Differences were considered statistically significant at $p<0.05$. 


\subsection{Experimental Results and Discussion}

(1) Reaction times: Table 2 shows the reaction times in the first (1 to 6 trials) and second half ( 7 to 12 trials) of the ATMT. The reaction times were delayed under the second half of the ATMT for most experiments, compared with that under the first half. The grand average $( \pm$ S.D.) values of the reaction times in all the experiments were $56.68 \pm 10.35 \mathrm{~s}$ in the first half vs. $60.01 \pm 9.26 \mathrm{~s}$ in the second half ( $p<0.05$ in the Wilcoxon signed rank sum test).

Table 2: Reaction Times in the First and Second Half Periods during the ATMT

\begin{tabular}{|c|c|c|c|}
\hline & & & \\
\hline Subj. & Times & $\begin{array}{c}\text { First half } \\
\text { (1 to } 6 \text { trials) }\end{array}$ & $\begin{array}{l}\text { Second half } \\
\text { (7 to } 12 \text { trials) }\end{array}$ \\
\hline & 1 & 48.24 & 48.51 \\
\hline$A$ & 2 & 46.59 & 49.07 \\
\hline & 3 & 46.29 & 52.35 \\
\hline & 1 & 71.29 & 78.72 \\
\hline$B$ & 2 & 66.42 & 71.83 \\
\hline & 3 & 63.86 & 67.24 \\
\hline & 1 & 49.18 & 56.08 \\
\hline$C$ & 2 & 46.83 & 51.86 \\
\hline & 3 & 55.36 & 62.13 \\
\hline & 1 & 75.11 & 61.12 \\
\hline$D$ & 2 & 60.00 & 61.86 \\
\hline & 3 & 50.97 & 59.36 \\
\hline Grand a & ge $\pm S . D$ & $56.68 \pm 10.35$ & $60.01 \pm 9.26 *$ \\
\hline
\end{tabular}

(2) Time domain analysis: We performed the Poincare plot as the time domain HRV analysis. The standard deviations $\left(\mathrm{SD}_{1}\right.$ and $\left.\mathrm{SD}_{2}\right)$ of data plots were computed in all experiments (Table 3). For most cases under the healthy conditions, the $\mathrm{SD}_{1}$ and $\mathrm{SD}_{2}$ values had a tendency to be decreased during the ATMT [Fig. 6(a) and (b)], compared to those before and after the test. These results presumably indicate the effect of fatigue or stress [24] induced by the test, activating the sympathetic nervous system. In contrast, the values after the ATMT suggest the increased parasympathetic activity with a relax state.

Figures 6(c)-(f) show the $\mathrm{SD}_{1}$ and $\mathrm{SD}_{2}$ values of Subjects $C$ and $D$; the blue, red, and green lines indicate the results of the first, second, and third experiments. Here, note that irregular values appeared under the unhealthy conditions. For example, the offset levels of $\mathrm{SD}_{1}$ and $\mathrm{SD}_{2}$ were remarkably lower under the unhealthy conditions than under the healthy conditions [Figs. 6(c)-(f): the second and third experiments in Subjects $C$ and $D$, respectively]; the $\mathrm{SD}_{2}$ value in Subject $C$ greatly increased after starting the ATMT [Fig. $6(d)]$. 
Table 3(a): Temporal Changes in $\mathrm{SD}_{1}$ Values of the Poincaré Plot during the Experiment

\begin{tabular}{|c|c|c|c|c|c|}
\hline \multirow{2}{*}{ Subj. } & \multirow[b]{2}{*}{ Times } & \multicolumn{4}{|c|}{ ATMT } \\
\hline & & Before & First half & Second half & After \\
\hline \multirow{3}{*}{$A$} & 1 & 15.24 & 8.07 & 10.08 & 11.26 \\
\hline & 2 & 21.57 & 10.07 & 11.98 & 18.15 \\
\hline & 3 & 18.88 & 14.60 & 13.79 & 17.16 \\
\hline \multirow{3}{*}{$B$} & 1 & 12.51 & 11.78 & 9.84 & 12.14 \\
\hline & 2 & 18.08 & 14.96 & 14.27 & 18.09 \\
\hline & 3 & 12.72 & 14.51 & 12.69 & 12.97 \\
\hline \multirow{3}{*}{$C$} & 1 & 36.79 & 37.19 & 34.05 & 39.75 \\
\hline & 2 & 12.00 & 8.17 & 11.38 & 11.52 \\
\hline & 3 & 43.95 & 37.63 & 47.04 & 55.99 \\
\hline \multirow{3}{*}{$D$} & 1 & 49.02 & 36.93 & 28.83 & 33.99 \\
\hline & 2 & 27.52 & 32.67 & 26.13 & 33.77 \\
\hline & 3 & 12.69 & 14.53 & 15.09 & 23.10 \\
\hline \multicolumn{2}{|c|}{$\begin{array}{c}\text { Grand aver- } \\
\text { age } \pm \text { S.D. }\end{array}$} & $23.42 \pm 13.04$ & $20.09 \pm 12.12$ & $19.60 \pm 11.80^{*, \# \#}$ & $23.99 \pm 14.03$ \\
\hline
\end{tabular}

${ }^{*}, p<0.05$ vs. before the ATMT; ${ }^{\#}, p<0.01$ vs. after the ATMT in the Tukey's post hoc test

Table 3(b): Temporal Changes in $\mathrm{SD}_{2}$ Values of the Poincaré Plot during the Experiment

\begin{tabular}{|c|c|c|c|c|c|}
\hline & \multicolumn{4}{|c|}{ ATMT } \\
\hline Subj. & Times & Before & First half & Second half & After \\
\hline \multirow{3}{*}{$A$} & 1 & 85.67 & 44.22 & 51.09 & 59.43 \\
\hline & 2 & 97.79 & 57.90 & 49.24 & 80.51 \\
\hline & 3 & 97.47 & 67.04 & 56.16 & 88.36 \\
\hline \multirow{3}{*}{$B$} & 1 & 52.45 & 43.27 & 37.23 & 59.60 \\
\hline & 2 & 60.55 & 33.41 & 34.62 & 64.36 \\
\hline & 3 & 60.32 & 33.58 & 34.93 & 61.93 \\
\hline \multirow{3}{*}{ C } & 1 & 77.54 & 62.69 & 77.78 & 100.55 \\
\hline & 2 & 26.72 & 61.44 & 45.14 & 47.47 \\
\hline & 3 & 61.82 & 65.64 & 72.69 & 89.90 \\
\hline \multirow{3}{*}{$D$} & 1 & 93.09 & 78.07 & 76.23 & 107.76 \\
\hline & 2 & 90.47 & 56.52 & 72.38 & 89.80 \\
\hline & 3 & 61.89 & 55.21 & 59.72 & 100.99 \\
\hline \multicolumn{2}{|c|}{$\begin{array}{l}\text { Grand aver- } \\
\text { age } \pm \text { S.D. }\end{array}$} & $72.15 \pm 21.79$ & $54.92 \pm 13.75^{\# \#}$ & $55.60 \pm 16.19^{\# \#}$ & $79.22 \pm 19.91$ \\
\hline
\end{tabular}

$p<0.01$ in the Friedman test \#\# $p<0.01$ vs. after the ATMT in the Tukey's post hoc test 
(a) $\mathrm{SD}_{1}$ (Ave.)

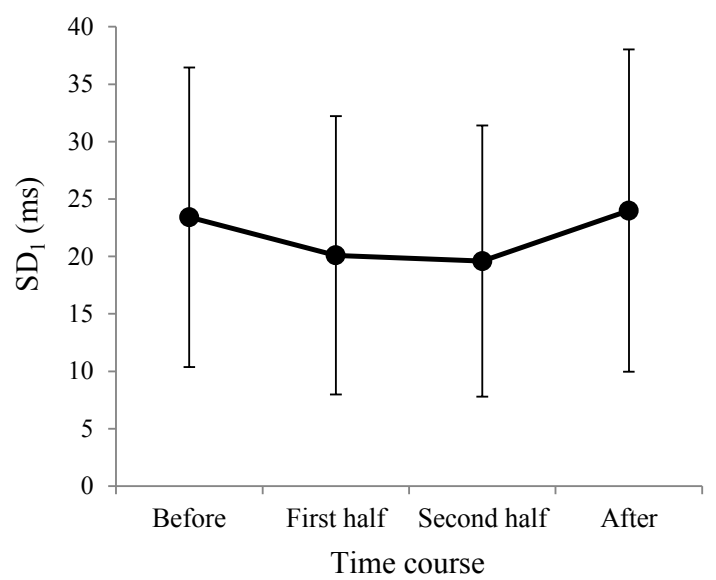

(c) $\mathrm{SD}_{1}$ (Sub. C)

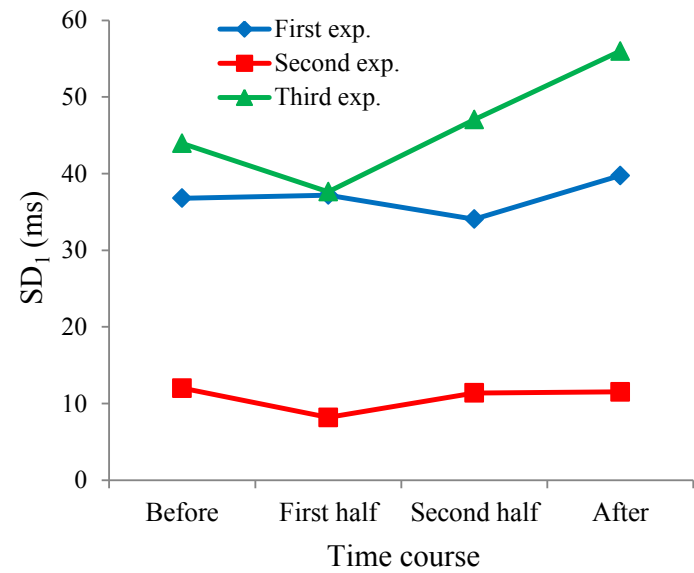

(e) $\mathrm{SD}_{1}(\mathrm{Sub} . D)$

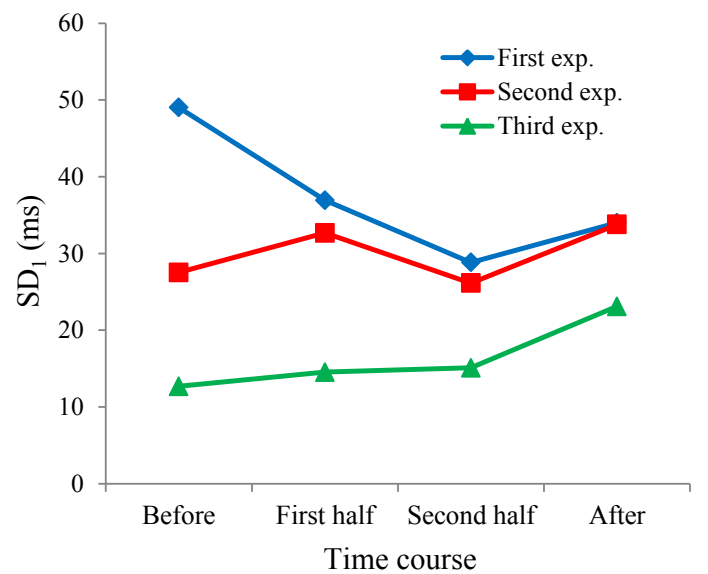

(b) $\mathrm{SD}_{2}$ (Ave.)

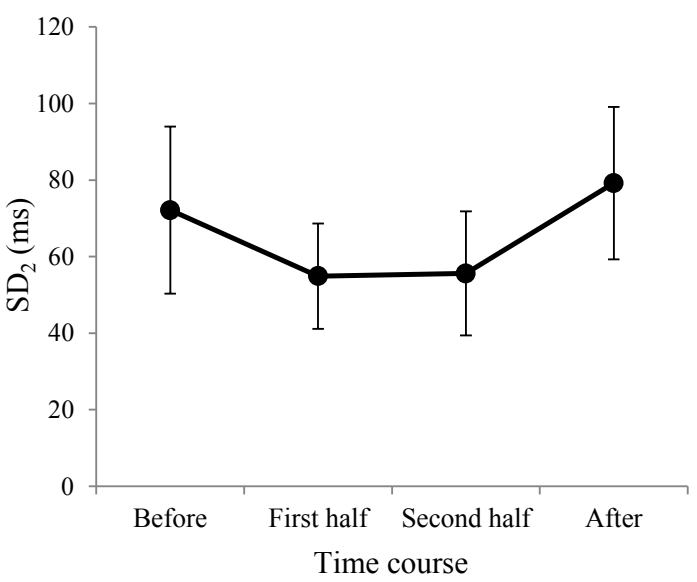

(d) $\mathrm{SD}_{2}$ (Sub. C)

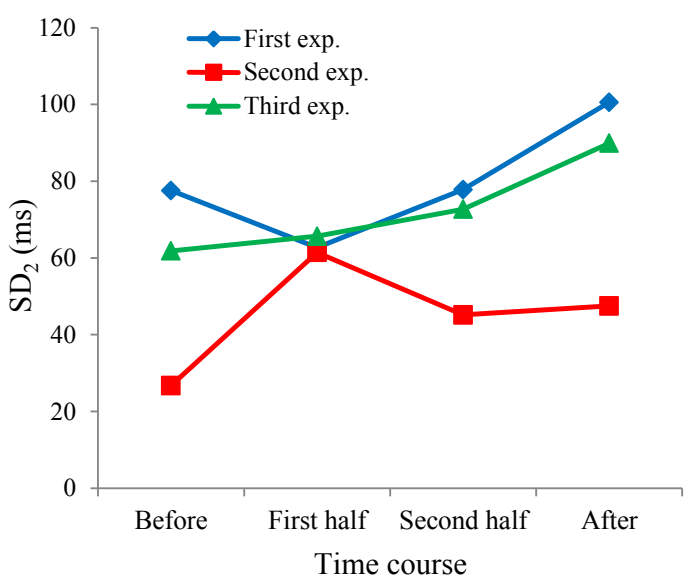

(f) $\mathrm{SD}_{2}$ (Sub. $D$ )

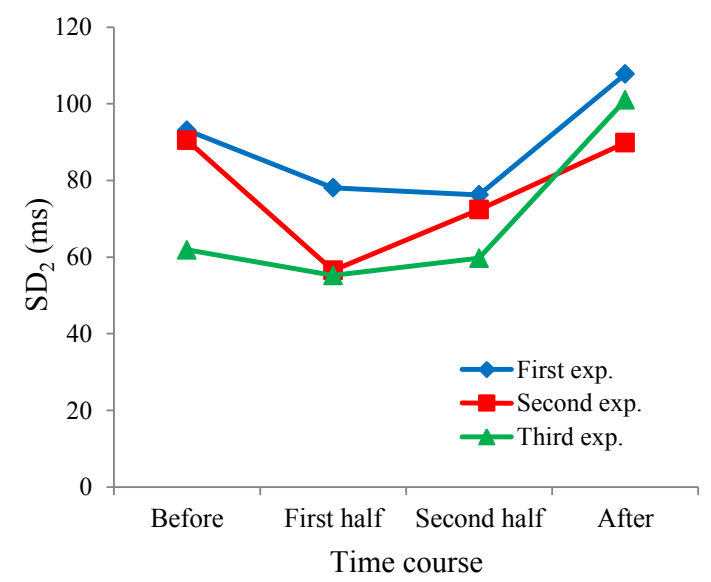

Figure 6: Temporal changes in parameters of the Poincare plot during the experiment: Grand averages ( \pm S.D.) across all experiments $\left[(a) \mathrm{SD}_{1}\right.$ and (b) $\left.\mathrm{SD}_{2}\right]$ and irregular cases [(c) $\mathrm{SD}_{1}$ and (d) $\mathrm{SD}_{2}$ in the second experiment of Subject $C$ (red lines); (e) $\mathrm{SD}_{1}$ and (f) $\mathrm{SD}_{2}$ in the third experiment of Subject $D$ (green lines)]. 
(3) Frequency domain analysis: We carried out the power spectrum analysis every subject (Table 4). The HF component reflecting the parasympathetic activity and the LF/HF component reflecting the sympathovagal balance were used for this analysis. The HF components under the healthy conditions had a tendency that the power spectrum values decreased during the ATMT, compared with those before and after the test [Fig. 7(a) and (b)]. This result means that the ATMT attenuated the parasympathetic activity. The HF component was then recovered after the test, indicating the increase of the parasympathetic activity [8] and a relax state. However, the LF/HF values were varied during each experiment, suggesting that the LF component contained various factors. For example, the deep breath during the stress task might have affected the LF component because the respiratory rate was not controlled for this study (i.e., natural respiration). Furthermore, the parasympathetic nervous activity will be considerably contained even in the LF component [25], although it mainly reflects the baroreceptor reflex and the sympathetic nervous activity.

Figures 7(c)-(f) represent the HF and LF/HF components of Subjects $C$ and $D$; the blue, red, and green lines mean the results of the first, second, and third experiments. The previous studies [21][23] showed the decreased HF and increased LF/HF components during the ATMT under the healthy conditions. In contrast, the specific feature of the HF and LF/HF components was observed under the unhealthy conditions. For example, the power spectrum values of the HF component were totally lower and the variance was smaller under the un-healthy condition [i.e., the second experiment in Subject $C$ and the third experiment of Subject $D$; Fig. 7(c) and (e)] than under the healthy conditions; those of the LF/HF compo-nent were the opposite responses [i.e., higher values in the unhealthy condition; Fig. 7(d) and (f)]. These results indicate that the sympathetic nervous activity was continuously acceler-ated under the unhealthy conditions with stress. Moreover, in Subject $C$, the power spectrum value of the LF/HF during the first half of the ATMT remarkably increased, compared with those in the other periods of the experiment [Fig. 7(d)]. The timefrequency analysis (e.g., the wavelet transform [26]) may efficiently detect a slight change in the heart function even under such abnormal conditions. 
Table 4(a): Temporal Changes in HF Values of the Power Spectrum Analysis during the Experiment

\begin{tabular}{|c|c|c|c|c|c|}
\hline \multirow[b]{2}{*}{ Subj. } & \multirow[b]{2}{*}{ Times } & \multicolumn{4}{|c|}{ ATMT } \\
\hline & & Before & First half & Second half & After \\
\hline \multirow{3}{*}{$A$} & 1 & 196.41 & 105.74 & 156.03 & 155.35 \\
\hline & 2 & 310.38 & 97.83 & 146.58 & 232.13 \\
\hline & 3 & 232.44 & 185.52 & 177.37 & 190.40 \\
\hline \multirow{3}{*}{$B$} & 1 & 172.22 & 142.09 & 128.57 & 184.20 \\
\hline & 2 & 245.79 & 203.17 & 163.58 & 265.93 \\
\hline & 3 & 196.31 & 198.54 & 189.54 & 192.98 \\
\hline \multirow{3}{*}{$C$} & 1 & 496.40 & 465.03 & 441.30 & 601.03 \\
\hline & 2 & 132.62 & 99.40 & 114.34 & 114.08 \\
\hline & 3 & 499.76 & 418.09 & 593.18 & 647.52 \\
\hline \multirow{3}{*}{$D$} & 1 & 590.73 & 359.12 & 289.52 & 405.08 \\
\hline & 2 & 362.23 & 374.65 & 341.07 & 427.72 \\
\hline & 3 & 148.06 & 171.24 & 169.81 & 274.43 \\
\hline \multicolumn{2}{|c|}{$\begin{array}{l}\text { Grand aver- } \\
\text { age } \pm S . D .\end{array}$} & $298.61 \pm 154.73$ & $235.03 \pm 132.37$ & $242.58 \pm 147.42^{*}, \#$ & $307.57 \pm 174.99$ \\
\hline
\end{tabular}

${ }^{*} p<0.05$ vs. before the ATMT; ${ }^{\#} p<0.05$ vs. after the ATMT in the Tukey's post hoc test

Table 4(b): Temporal changes in LF/HF Values of the Power Spectrum Analysis during the Experiment

\begin{tabular}{|c|c|c|c|c|c|}
\hline \multicolumn{2}{|c|}{} & \multicolumn{5}{|c|}{ ATMT } \\
\hline Subj. & Times & Before & First half & Second half & After \\
\hline \multirow{4}{*}{$A$} & 1 & 5.33 & 4.38 & 3.64 & 4.97 \\
\cline { 2 - 7 } & 2 & 3.34 & 3.31 & 3.62 & 3.70 \\
\cline { 2 - 7 } & 3 & 4.64 & 4.54 & 3.83 & 5.81 \\
\hline \multirow{4}{*}{$B$} & 1 & 4.27 & 2.09 & 2.65 & 3.50 \\
\cline { 2 - 7 } & 2 & 3.34 & 1.58 & 1.96 & 1.35 \\
\cline { 2 - 7 } & 3 & 2.94 & 1.92 & 1.59 & 2.26 \\
\hline \multirow{4}{*}{$C$} & 1 & 1.95 & 1.38 & 1.76 & 1.48 \\
\cline { 2 - 7 } & 2 & 1.82 & 4.45 & 2.32 & 2.84 \\
\cline { 2 - 6 } & 3 & 0.95 & 1.63 & 1.03 & 0.83 \\
\hline \multirow{4}{*}{$D$} & 1 & 1.45 & 1.58 & 2.41 & 2.15 \\
\cline { 2 - 6 } & 2 & 2.31 & 1.76 & 2.39 & 2.09 \\
\cline { 2 - 6 } & 3 & 3.25 & 3.25 & 3.12 & 3.34 \\
\hline \multicolumn{2}{|c|}{ Grand aver- } & $2.96 \pm 1.33$ & $2.66 \pm 1.25$ & $2.53 \pm 0.88$ & $2.86 \pm 1.49$ \\
\hline
\end{tabular}


(a) HF (Ave.)

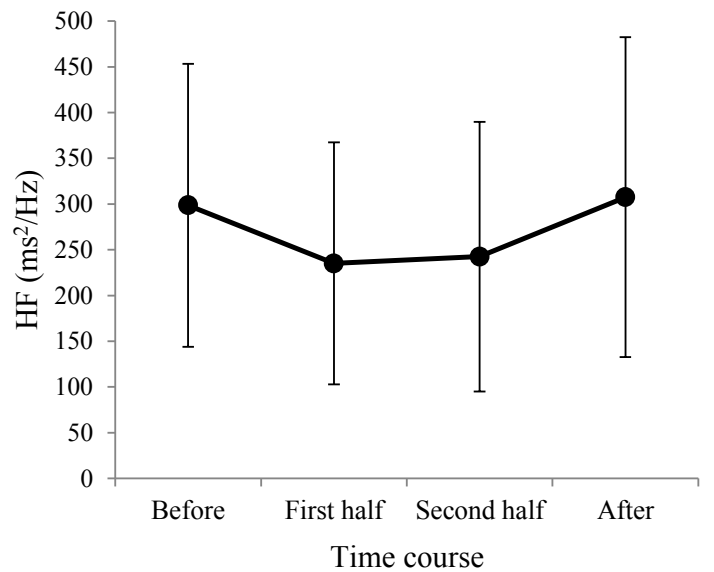

(c) HF (Sub. C)

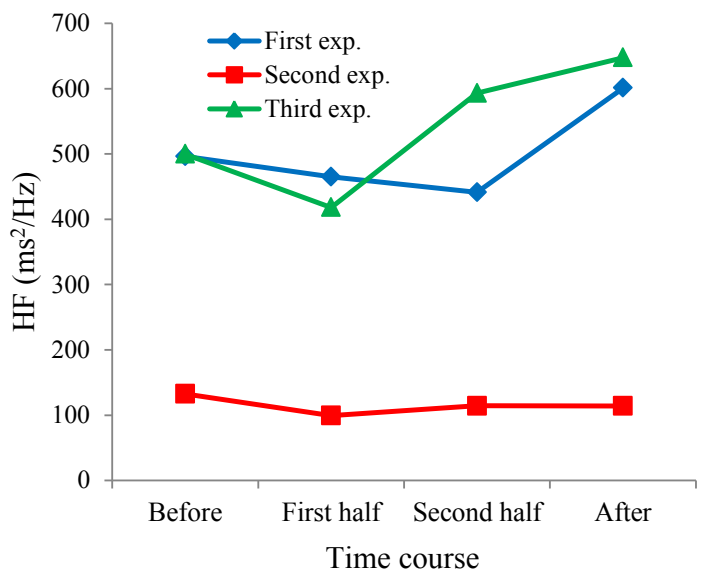

(e) HF (Sub. D)

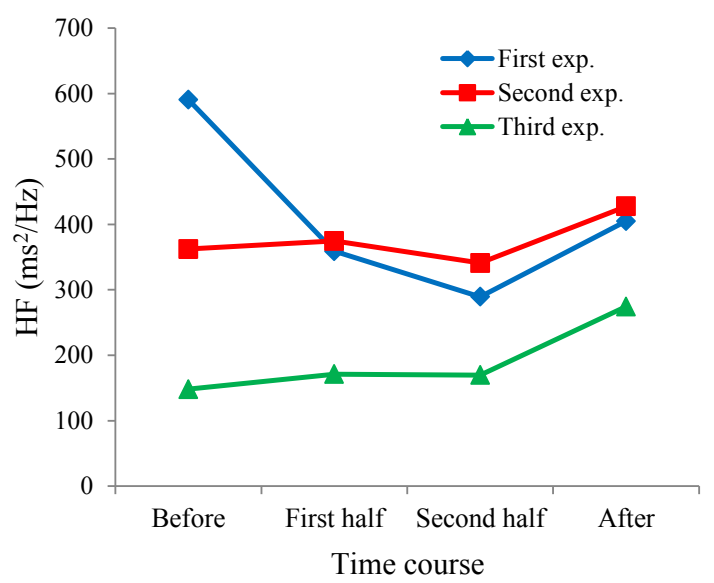

(b) LF/HF (Ave.)

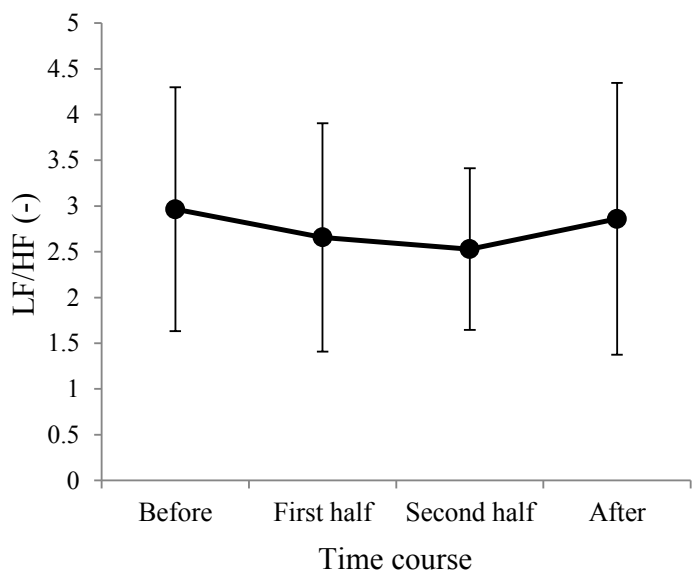

(d) LF/HF (Sub. C)

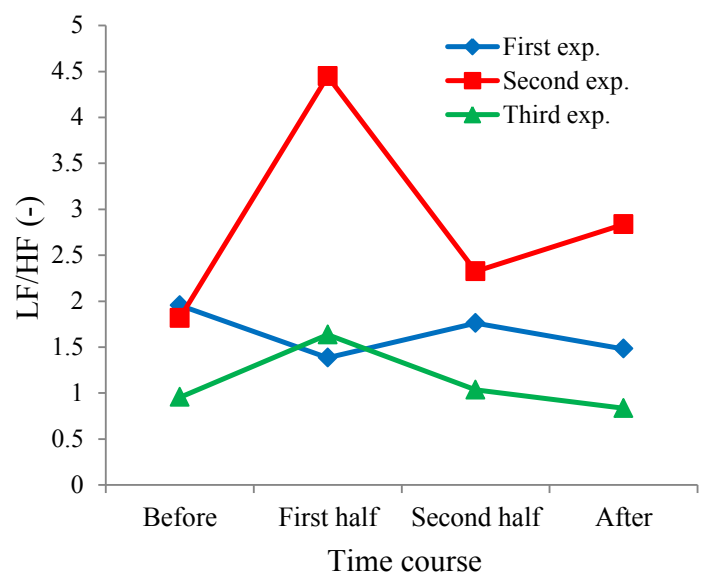

(f) LF/HF (Sub. D)

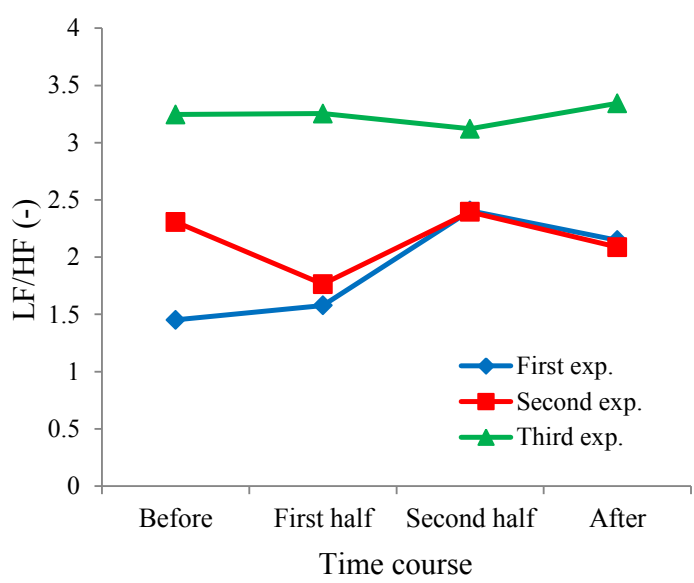

Figure 7: Temporal changes in the power spectrum values. Grand averages ( \pm S.D. $)$ across all experiments: (a) HF and (b) LF/HF components; the irregular cases in Subjects $C$ [(c) HF and (d) HF/LF in the second experiment (red lines)] and $D$ [(e) HF and (f) HF/LF in the third experiment (green lines)]. 
(4) Relationship between the time and frequency analyses: The correlation coefficient (Table 5) was computed to clarify the relationship between the evaluation indices in the time and frequency domains. The HF component was tightly associated with the $\mathrm{SD}_{1}$ value; the high correlation will be due to the fact that both the indices reflect the parasympathetic activity. In the other correlation coefficients, there were individual differences. In special, the unusual data (i.e., the second experiment in Subject $C$ and the third experiment in Subject $D$ ) varied the correlation coefficients. For Subject $C$, the correlation coefficient between the HF component and $\mathrm{SD}_{2}$ was lower under the unhealthy condition than under the healthy condition. By contrast, for Subject $D$, the correlation coefficient between the HF component and $\mathrm{SD}_{2}$ was higher under the unhealthy condition than under the healthy condition. These results suggest that the correlation under the unusual condition has a specific feature when compared with the normal data.

Table 5: Correlation Coefficients between the HRV Indices

in the Time and Frequency Domains

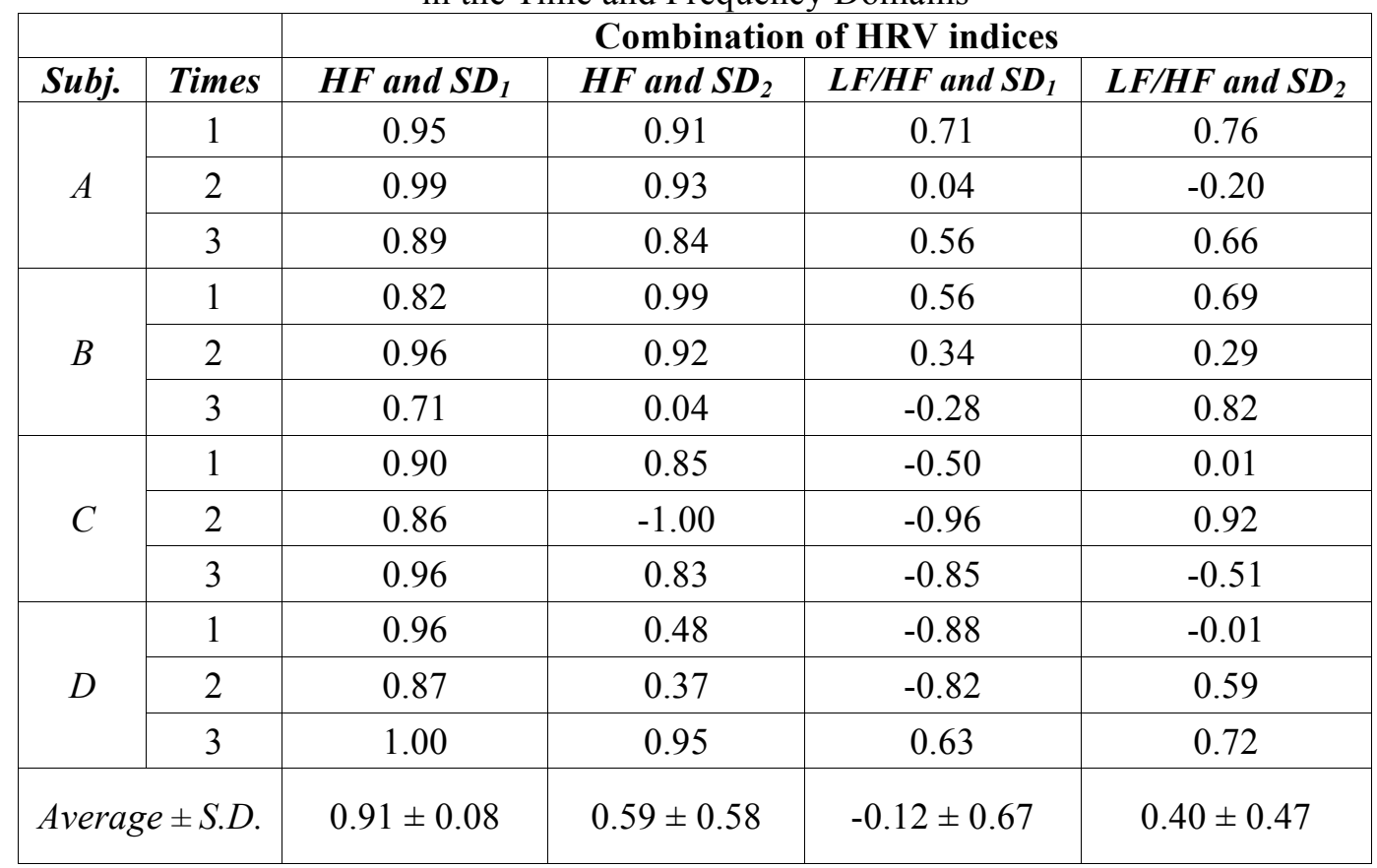

\section{User Interface for HRV Analysis}

To explore the possibility as a home-healthcare application, we created a user interface based on the Poincare plot (Visual Basic 6.0) to easily check the daily condition of heart functions with temporal changes. Because the Poincaré plots of the multiple time-course data are added to a figure panel [Fig. 8(a)], they can reveal the daily changes of the autonomic nervous activity. The RR intervals after the axis rotation can be also visualized [Fig. 8(b)]. The ellipses were illustrated from the $\mathrm{SD}_{1}$ and $\mathrm{SD}_{2}$ values [Fig. 8(c)]. Temporal changes in the features of the time domain $\mathrm{HRV}$ analysis (i.e., $\mathrm{SD}_{1}, \mathrm{SD}_{2}, \mathrm{SD}_{1} / \mathrm{SD}_{2}$, and the area of an ellipse) can be compared each other, as numerical data [Fig. 8(d) and (e)].

The second study (i.e., Section 3) had the limitation in the number of experimental times (i.e., three times every subject); however, by using the user inter face as a home healthcare system, the continuous measurement with the HRV analysis (i.e., the cumulated data) could increase the 
accuracy and detect a slight temporal change at an early stage of diseases. The artificial intelligence and machine learning [27][28] can be also implanted into this interface, in order to judge the abnormal or unusual state from the experimental data and the HRV analyses.

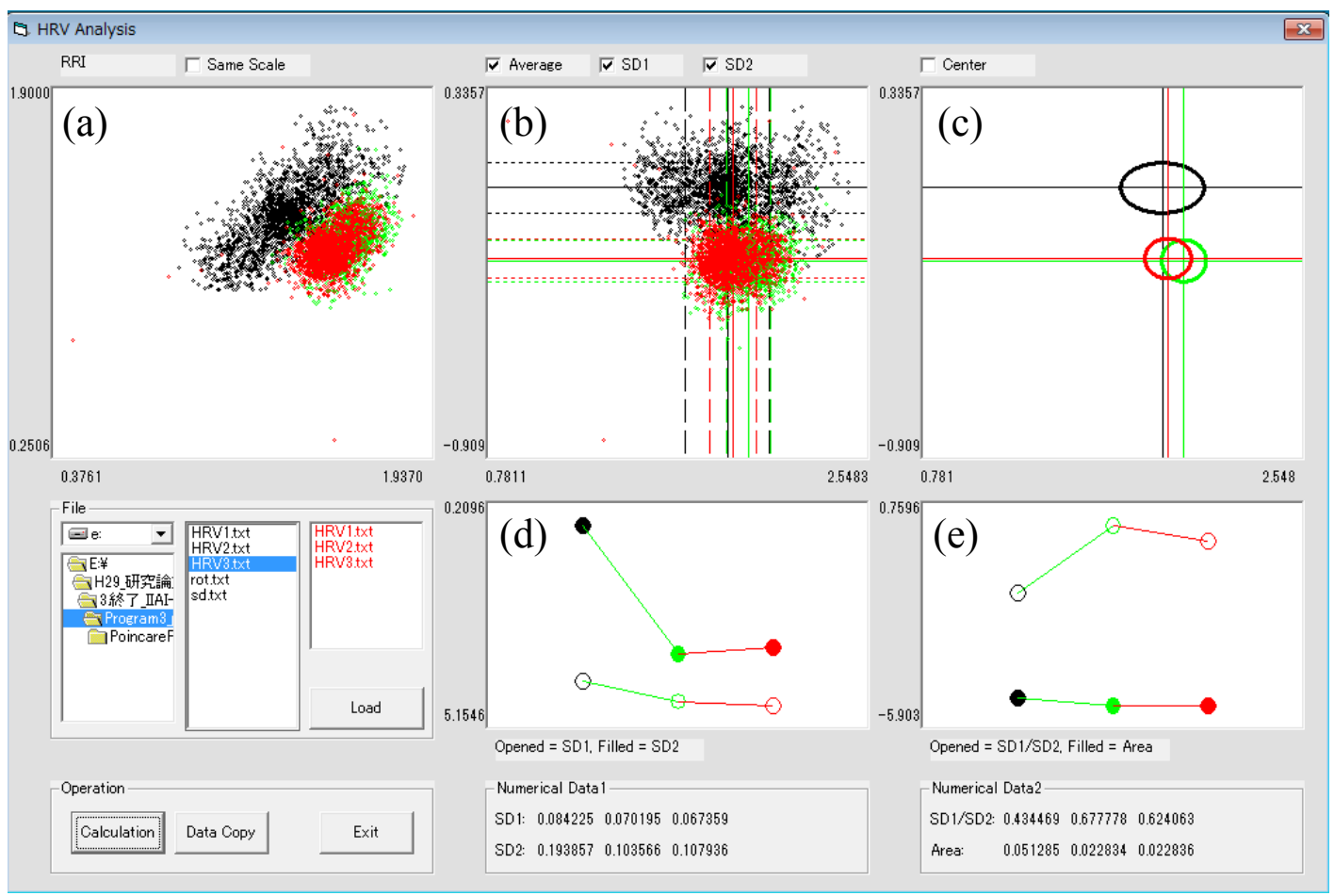

Figure 8: The created user interface for analyzing the temporal changes in the Poincare plot. (a) The Poincaré plots of the multiple time-course data. (b) The RR intervals after the axis rotation; Dotted lines: standard deviations. (c) The ellipses illustrated from the $\mathrm{SD}_{1}$ and $\mathrm{SD}_{2}$ values. Temporal changes in (d) $\mathrm{SD}_{1}$ and $\mathrm{SD}_{2} ;$ (e) $\mathrm{SD}_{1} / \mathrm{SD}_{2}$ and the areas of ellipses.

\section{Conclusions}

We explored for the analytical method to simply and automatically catch the abnormalities or temporal changes in heart function. To detect the ST depression from abnormal ECG data, the optimal threshold value was determined by the modified cross-validation analysis based on the correlation coefficient between ECG signals on the template and other diseases. The optimal threshold of the correlation coefficient existed (i.e., around 0.8 for this study); it was little affected by the type of interpolations and window sizes for a normalized template. If it is important to reduce the computation time in online analysis for smartphone or tablet applications, the small data size will be preferred.

Next, the HRV analysis in the time or frequency domain was performed during the ATMT, in order to assess the temporal change in individual's function of the heart. The Poincare plot in the time domain analysis revealed that the $\mathrm{SD}_{1}$ and $\mathrm{SD}_{2}$ values depend on the state of tension and concentration during the ATMT, suggesting the unhealthy conditions. The HF component in the frequency domain analysis expressed the temporal changes in the healthy and unhealthy conditions during the test, mainly reflecting the parasympathetic activity. The high relationship between the $\mathrm{SD}_{1}$ and $\mathrm{HF}$ component suggests the similar evaluation indices. As shown in Fig. 8, the evaluation indices for the cardiac analysis with daily cumulative 
data could be applicable for a home healthcare system to simply and automatically check the temporal changes in heart functions.

\section{Acknowledgement}

This study was partly funded by a Grant-in-Aid for Scientific Research (C) from Japan Society for the Promotion of Science (KAKENHI 25330171; 17K01297).

\section{References}

[1]D. Mozaffarian, et al., "Executive summary: Heart disease and stroke statistics-2016 update a report from the American Heart Association," Circulation, vol. 133, no. 4, 2016, pp. $447-454$.

[2] S.J. Schang and C.J. Pepine, "Transient asymptomatic ST segment depression during daily activity," The American journal of cardiology, vol. 39, no. 3, 1977, pp. 396-402.

[3] J.E. Deanfield, M. Shea, P. Ribiero, et al., "Transient ST-segment depression as a marker of myocardial ischemia during daily life," The American journal of cardiology, vol. 54, no. 10,1984 , pp. $1195-1200$.

[4]K. Bawa and P. Sabharwal, "ECG signal fibrillation classification on android platform: A survey approach," International Journal of Emerging Science and Engineering, vol. 2, no. 7, 2014, pp. 1-4.

[5]F. Jager, G.B. Moody, and R.G. Mark, "Detection of transient ST segment episodes during ambulatory ECG monitoring," Comput. Biomed. Res., vol. 31, no. 5, 1998, pp. 305322.

[6] J.A. Walsh, et al., "Novel wireless devices for cardiac monitoring," Circulation, vol. 130, no. 7, 2014, pp. 573-581.

[7] M.P. Tulppo, et al., "Quantitative beat-to-beat analysis of heart rate dynamics during exercise," American Journal of Physiology-Heart and Circulatory Physiology, vol. 271, no. 1, 1996, pp. H244-H252.

[8] J. Altimiras, "Understanding autonomic sympathovagal balance from short-term heart rate variations. Are we analyzing noise?," Comparative Biochemistry and Physiology Part A: Molecular \& Integrative Physiology, vol. 124, no. 4, 1999, pp. 447-460.

[9] K. Mizuno and Y. Watanabe, "Utility of an advanced trail making test as a neuropsychological tool for an objective evaluation of work efficiency during mental fatigue," In: Fatigue Science for Human Health, Springer Japan, 2008, pp. 47-54.

[10] M.I. Owis, A.H. Abou-Zied, A.B. Youssef, and Y.M. Kadah, "Study of features based on nonlinear dynamical modeling in ECG arrhythmia detection and classification," IEEE transactions on Biomedical Engineering, vol. 49, no. 7, 2002, pp. 733-736.

[11] W. Zong, M. Saeed, and T. Heldt, "A QT interval detection algorithm based on ECG curve length transform," Computers in Cardiology, 2006, pp. 377-380.

[12] P. De Chazal, M. O'Dwyer, and R.B. Reilly, "Automatic classification of heartbeats using ECG morphology and heartbeat interval features," IEEE Transactions on Biomedical Engineering, vol. 51, no. 7, 2004, pp. 1196-1206.

[13] T. Syeda-Mahmood, D. Beymer, and F. Wang, "Shape-based matching of ECG recordings," In Engineering in Medicine and Biology Society, 29th Annual International Conference of the IEEE, 2007, pp. 2012-2018.

[14] J. Presedo, J. Vila, M. Delgado, S. Barro, F. Palacios, and R. Ruiz, "A proposal for the fuzzy evaluation of ischaemic episodes," in Computers in Cardiology. Los Alamitos, 
CA: IEEE Comput. Soc. Press, 1995, pp. 709-712.

[15] H.M. Rai, A. Trivedi, and S. Shukla, "ECG signal processing for abnormalities detection using multi-resolution wavelet transform and Artificial Neural Network classifier," Measurement, vol. 46, no. 9, 2013, pp. 3238-3246.

[16] D.H. Lee, J.W. Park, J. Choi, A. Rabbi, and R. Fazel-Rezai, "Automatic detection of electrocardiogram ST segment: Application in ischemic disease diagnosis," International Journal of Advanced Computer Science and Applications, 2013, vol. 4, no. 2.

[17] J. García, L. Sornmo, S. Olmos, and P. Laguna, "Automatic detection of ST-T complex changes on the ECG using filtered RMS difference series: application to ambulatory ischemia monitoring," IEEE Transactions on Biomedical Engineering, vol. 47, no. 9, 2000, pp. 1195-1201.

[18] C.C. Chiu, T.H. Lin, and B.Y. Liau, "Using correlation coefficient in ECG waveform for arrhythmia detection," Biomedical Engineering: Applications, Basis and Communications, vol. 17, no. 03, 2005, pp. 147-152.

[19] C.T. Lin, et al., "An intelligent telecardiology system using a wearable and wireless ECG to detect atrial fibrillation," IEEE Transactions on Information Technology in Biomedicine, vol. 14, no. 3, 2010, pp. 726-733.

[20] K. Sternickel, "Automatic pattern recognition in ECG time series," Computer methods and programs in biomedicine, vol. 68, no. 2, 2002, pp. 109-115.

[21] M. Tanaka, et al., "Autonomic nervous alterations associated with daily level of fatigue," Behavioral and Brain Functions, 2011, vol. 7, 46.

[22] J.S. Stamler, M.E. Goldman, J. Gomes, D. Matza, and S.F. Horowitz, "The effect of stress and fatigue on cardiac rhythm in medical interns," Journal of electrocardiology, vol. 25 , no. 4,1992 , pp. 333-338.

[23] M. Tanaka, K. Mizuno, S. Tajima, T. Sasabe, and Y. Watanabe, "Central nervous system fatigue alters autonomic nerve activity," Life sciences, vol. 84, no. 7, 2009, pp. 235239.

[24] R. Orsila, et al., "Perceived mental stress and reactions in heart rate variability - a pilot study among employees of an electronics company," International Journal of Occupational Safety and Ergonomics, vol. 14, no. 3, 2008, pp. 275-283.

[25] M.S. Houle and G.E. Billman, "Low-frequency component of the heart rate variability spectrum: a poor marker of sympathetic activity," American Journal of Physiology-Heart and Circulatory Physiology, vol. 276, no. 1, 1999, pp. H215-H223.

[26] K. Kashihara, T. Kawada, M. Sugimachi, and K. Sunagawa, "Wavelet-based system identification of short-term dynamic characteristics of arterial baroreflex," Annals of Biomedical Engineering, vol. 37, no. 1, 2009, pp. 112-128.

[27] K. Kashihara, M. Ito, and M. Fukumi, "Automatic system to remove unpleasant images detected by pupil-size changes," International Journal of Computer Science Issues, vol. 9, no. 1, 2012, pp. 68-73.

[28] K. Kashihara, "A brain-computer interface for potential nonverbal facial communication based on EEG signals related to specific emotions," Frontiers in Neuroscience, vol. 8, no. 244, 2014, pp. 1-12. 